\title{
Active Cancellation of Probing in Dipole Arrays including Mutual Coupling Effect
}

\author{
N. Bala Ankaiah, Hema Singh, R. M. Jha \\ Centre for Electromagnetics (CEM) \\ CSIR - National Aerospace Laboratories \\ Bangalore 560017 \\ Email: hemasingh@nal.res.in,jha@nal.res.in
}

\begin{abstract}
A modified improved LMS algorithm is used for active cancellation of probing sources in arbitrary linear dipole array. The mutual coupling is included in the formulation of the received signal vector and hence weight adaptation. The signal environment consists of the multiple narrowband uncorrelated desired and probing sources. The dipole array performance in adaptive nulling is analyzed in terms of output signal-tointerference-noise ratio (SINR) and adapted pattern. It is shown that parallel-in-echelon dipole array is able to maintain multilobe beamforming with accurate and deep nulls towards each probing source.

Index Terms - Adapted pattern, dipole array, modified improved LMS algorithm, mutual coupling, output SINR.
\end{abstract}

\section{INTRODUCTION}

The capability of an efficient phased array system is to maintain gain towards desired directions and simultaneously cancelling the hostile sources attempting to probe the array system. This is true even when array does not have any apriori information regarding arrival angles, power level, or frequency of the impinging signals. The array performance depends on the array design parameters including the antenna element radiation pattern, geometrical configuration, etc. The requirement of limited antenna aperture makes the design compact, with antenna elements placed close to each other. This gives rise to mutual coupling effect, which in turn affects the array response. The performance gets degraded in terms of direction-of-arrival (DOA) estimation, output SINR and probe suppression. This effect makes essential to compensate the mutual coupling effect towards optimized array performance [1]. The antenna terminal voltages are decoupled using the mutual impedance matrix (MIM) [2], and direct-data domain $\left(D^{3}\right)$ algorithm towards adaptive nulling [3]. These decoupled open-circuited voltages are used in weight adaptation for suppressing unwanted signals. Another method to extract desired signal from a given signal scenario is to employ transformation matrix to the measured voltages at the antenna elements [4], so as to generate set of induced voltages for uniform linear array of isotropic elements. Then a $\mathrm{D}^{3}$ least square algorithm is used to extract information about the desired signals and generate adapted pattern. This method is reported to cater to the problems of mutual coupling effect and strong near-field scatterers for uniform and non-uniform phased arrays. The mutual coupling compensation can also be achieved by using space-time adaptive processing (STAP), where the nulling performance of adaptive arrays improves with increase in number of antenna elements keeping array aperture fixed [5]. This is due to increase in available degrees of freedom for the array.

In the present paper, the modified improved LMS algorithm is used for adaptive nulling in linear dipole array including mutual coupling effect. An arbitrary signal environment consisting of multiple narrowband uncorrelated desired and probing sources are considered. It is assumed that DOA of the signals is known a priori. A mutual impedance matrix consisting of self and mutual impedance is included in the received field vector. The performance of dipole array is analyzed in terms of output SINR and adapted pattern. It is shown that dipole array is able to maintain multilobe beamforming with accurate and deep nulls towards each probing source even in the presence of mutual coupling effect.

\section{THEORETICAL BACKGROUND}

The radiation characteristics of phased array depend on the array design parameters. The geometric configuration and inter-element spacing controls the mutual coupling effect in between the elements. The coupling effect changes the array impedance and hence the received signal vector and array response. This in turn varies the array correlation matrix and the optimum weights used for antenna element excitation. Thus it is not ambiguous to state that the adapted pattern of phased array for a given signal scenario depends on the mutual coupling effect.

The array response towards the signal incident at an angle of $\theta$ is given by

$$
S=\sum_{i=1}^{N} I_{i} e^{-j(k d \cos \theta+\alpha)} .
$$

where $k$ is the wave number, $d$ is the inter-element spacing, $I_{i}$ and $\alpha$ are the amplitude and phase excitations, respectively. The corresponding electric field associated with the incident signal is expressed as

$$
E_{i}(\theta, \phi)=f(\theta, \phi) \sum_{i=1}^{N} I_{i} e^{-j(k d \cos \theta+\alpha)} .
$$

$f(\theta, \phi)$ is the radiation pattern of a dipole given by

$$
f(\theta, \phi)=\frac{j \eta_{o} k I_{o} l e^{-j k r} \sin \theta[2 \cos (k h \cos \theta)]}{4 \pi R} .
$$


where $\eta_{o}$ is the free space characteristic impedance, $l$ is the dipole length, $I_{o}$ is the current fed, $R$ is the far-field distance and $h$ is the dipole height above the ground plane.

The radiation pattern of the dipole array is given by

$$
\left|E_{i}(\theta, \phi)\right|=|f(\theta, \phi)| \cdot|S| .
$$

The modified improved LMS algorithm is employed to estimate optimum antenna weights. The algorithm is based on the principle of iterative calculation of weights, maintaining the Toeplitz structure of signal covariance matrix. The antenna weights for a linear dipole array are iteratively computed as

$$
W(n+1)=P[W(n)-\mu g(W(n))]+(I-P)
$$

where, the projection operator is

$$
P=I-\frac{S_{o_{1}} S_{O_{1}}^{H}}{S_{O_{1}}^{H} S_{o_{1}}}-\frac{S_{o_{2}} S_{o_{2}}^{H}}{S_{O_{2}}^{H} S_{o_{2}}}-\ldots-\frac{S_{o_{p}} S_{o_{p}}^{H}}{S_{o_{p}}^{H} S_{o_{p}}} .
$$

$S_{o_{1}}, S_{o_{2}}, \ldots, S_{o_{p}}$ represent array manifold to $p$ desired signals impinging the array at different angles. The gradient $g$ in (5) is given by

$$
g[W(n)]=2 \widetilde{R}(n+1) W(n)
$$

with $\widetilde{R}(n+1)=\frac{1}{n+1}[n \widetilde{R}(n)+\hat{R}(n+1)]$

For uncorrelated impinging signals, the Toeplitz form of $\hat{R}(n)$ in the presence of mutual coupling, is expressed as

$$
\hat{R}(n)=Z_{o}^{-1}\left[\begin{array}{llll}
\hat{r}_{o}(n) & \hat{r}_{1}(n) & \ldots & \hat{r}_{(L-1)}(n) \\
\hat{r}_{1}^{*}(n) & . & & \\
\vdots & : & & \\
\hat{r}_{(L-1)}{ }^{*}(n) & & & r_{o}(n)
\end{array}\right] .
$$

The impedance matrix $Z_{\mathrm{o}}$ consists of both self impedance and mutual impedance components. Once the optimum weights are obtained, the adapted pattern for a given signal scenario is given by

$$
\text { Pattern }(\mathrm{dB})=20 \log _{10}(G)
$$

with $G=W_{\text {optimum }} . S^{\prime}$.

$$
\begin{gathered}
S^{\prime}=\left[f_{1}\left(\theta^{\prime}, \phi^{\prime}\right) \cdot e^{-j k d \cdot 0 \cdot \cos \theta^{\prime}} f_{2}\left(\theta^{\prime}, \phi^{\prime}\right) \cdot e^{-j k d \cdot 1 \cdot \cos \theta^{\prime}}\right. \\
\left.\ldots f_{N}\left(\theta^{\prime}, \phi^{\prime}\right) \cdot e^{-j k d \cdot(N-1) \cdot \cos \theta^{\prime}}\right] .
\end{gathered}
$$

The output signal-to-interference-noise ratio is expressed as

$$
S I N R=\frac{W^{H} R W-W^{H} R_{n} W}{W^{H} R_{n} W}
$$

where,

$$
R=x^{H} . x \text {. }
$$

$W$ is the optimum weight obtained from (5) for a given signal scenario, $R_{n}$ is the noise correlation matrix.

\section{RESULTS AND DISCUSSION}

A 16-element uniform linear parallel-in-echelon dipole array is considered. The dipole length is taken as halfwavelength with $h=0.25 \lambda$. The inter-element spacing is $0.484 \lambda$. In adapted pattern, the desired sources are shown as green arrows while probing sources are represented by red arrows. Figure 1 shows the output noise power of 16-element linear parallel-in-echelon dipole array with one desired signal $\left(90^{\circ} ; 1\right)$ and two probing sources $\left(35^{\circ}, 145^{\circ} ; 100\right.$ each $)$. The output noise power of parallel-in-echelon array is compared with that of side-by-side dipole array. The output noise power of parallel-in-echelon array is lower than that of side-by-side configuration. Moreover, it may be observed that the performance of parallel-in-echelon array without mutual coupling effect is better than that of arrays with mutual coupling. Next, the output SINR of 16-element linear parallelin-echelon dipole array is analyzed (Fig. 2). The signal scenario consists of one desired signal $\left(90^{\circ} ; 1\right)$ and two probing sources $\left(45^{\circ}, 135^{\circ} ; 100\right.$ each). The performance of parallel-in-echelon degrades a little on inclusion of mutual coupling effect.

Figure 3 shows the adapted pattern of 16-element linear parallel-in-echelon dipole array with two desired signals $\left(40^{\circ}\right.$, $90^{\circ} ; 1$ each) and one probing source $\left(150^{\circ} ; 100\right)$. It is apparent that the array maintains the gain towards desired sources and at the same time null is placed towards the probing source without any distortion in the mainlobes. The number of probing sources are next increased to two $\left(80^{\circ}, 100^{\circ} ; 100\right.$ each) keeping the two desired signals $\left(40^{\circ}, 140^{\circ} ; 1\right.$ each). The adapted pattern of 16-element linear parallel-in-echelon dipole array is shown in Fig. 4. The pattern is as per the expectations.

Next the number of desired signals is increased to three $\left(40^{\circ}, 90^{\circ}, 140^{\circ} ; 1\right.$ each $)$ and keeping two probing sources $\left(70^{\circ}\right.$, $110^{\circ} ; 100$ each). The resultant adapted pattern is shown in Fig. 5. Again, each of two probing sources is suppressed without any distortion in the three main lobes towards each of the desired signals. The overall pattern remains undisturbed.

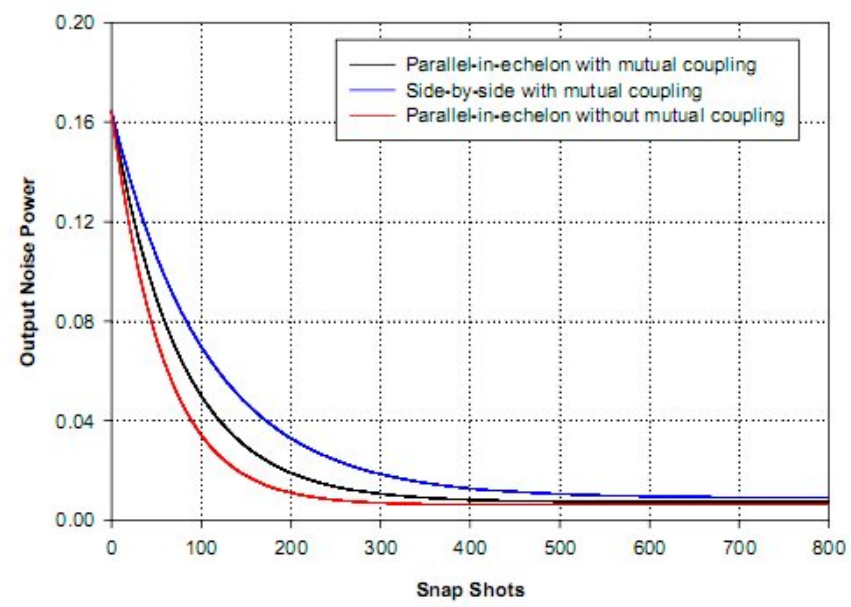

Fig. 1. Output noise power of 16-element linear parallel-inechelon dipole array. One desired signal $\left(90^{\circ} ; 1\right)$ and two probing sources $\left(35^{\circ}, 145^{\circ} ; 100\right.$ each $)$. 


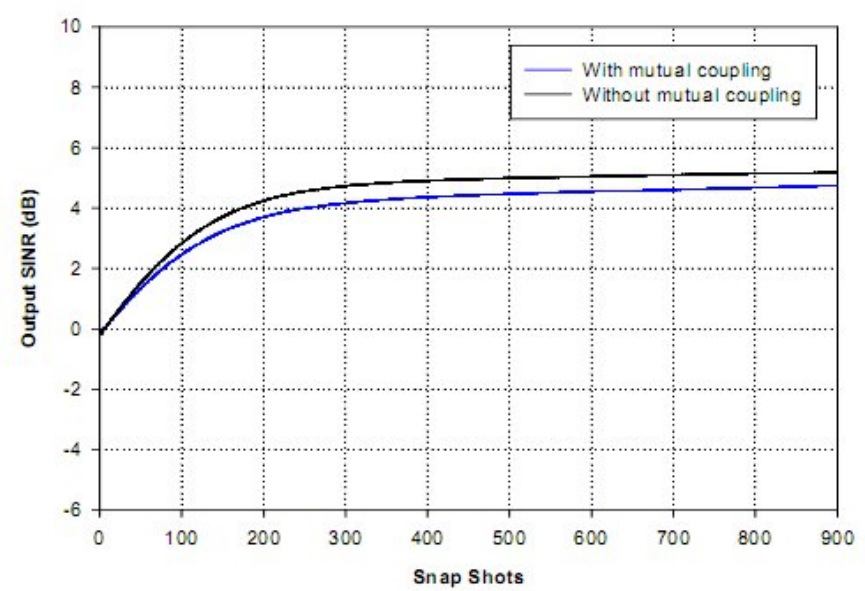

Fig. 2. Output SINR of 16-element linear parallel-in-echelon dipole array. One desired signal $\left(90^{\circ} ; 1\right)$ and two probing sources $\left(45^{\circ}, 135^{\circ} ; 100\right.$ each $)$.

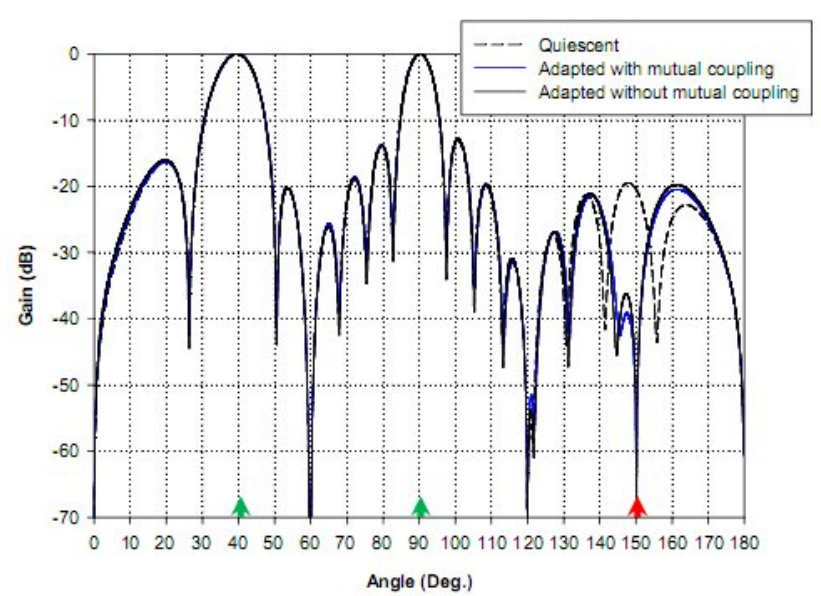

Fig. 3. Adapted pattern of 16-element linear parallel-in-echelon dipole array. Two desired signals $\left(40^{\circ}, 90^{\circ} ; 1\right.$ each) and one probing source $\left(150^{\circ} ; 100\right)$.

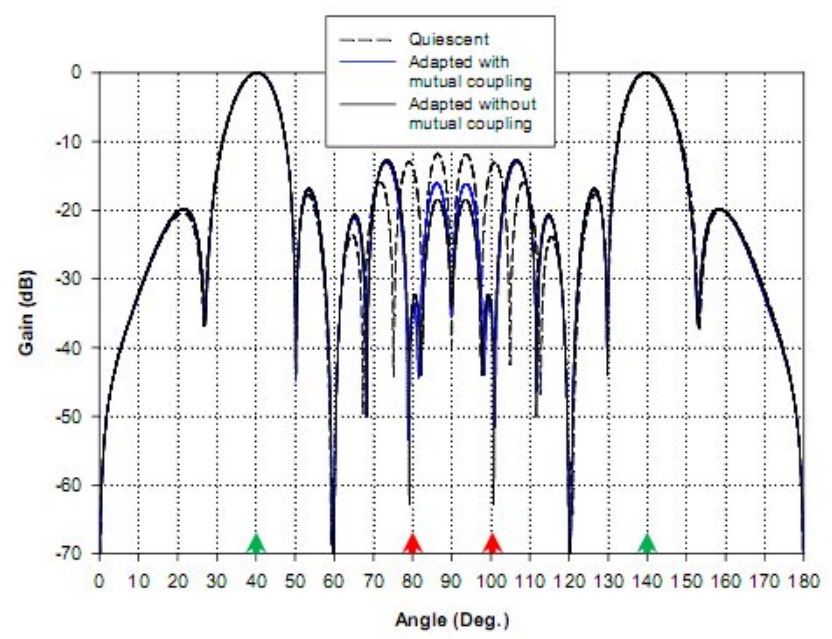

Fig. 4. Adapted pattern of 16-element linear parallel-in-echelon dipole array. Two desired signals $\left(40^{\circ}, 140^{\circ} ; 1\right.$ each $)$ and two probing sources $\left(80^{\circ}, 100^{\circ} ; 100\right.$ each).

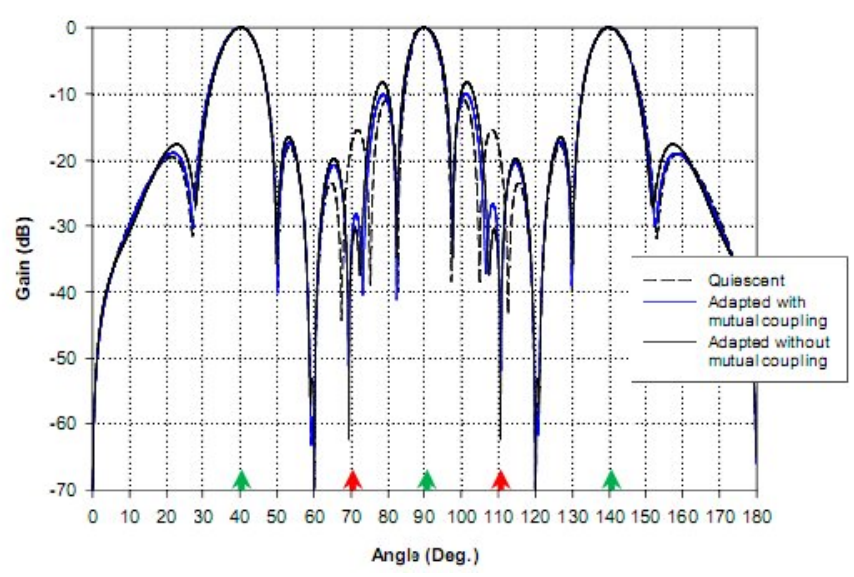

Fig. 5. Adapted pattern of 16-element linear parallel-in-echelon dipole array. Three desired signals $\left(40^{\circ}, 90^{\circ}, 140^{\circ} ; 1\right.$ each) and two probing sources $\left(70^{\circ}, 110^{\circ} ; 100\right.$ each).

\section{CONCLUSION}

This paper establishes the efficacy of modified improved LMS algorithm in dipole array for active cancellation of multiple probing sources, and simultaneously maintaining gain towards each desired source. The weight adaptation and hence the adapted pattern depends on the array configuration, signal scenario, and the mutual coupling effect. It is shown that the adaptive nulling performance is well maintained for an arbitrary signal environment and even in the presence of mutual coupling effect. In adapted pattern, the nulls are placed accurately towards each of probing directions without any distortion in the mainlobe and sidelobe levels. This demonstrates the capability of the algorithm in actively reducing the detectability of phased array.

\section{REFERENCES}

[1] H. T. Hui, "A practical approach to compensate for the mutual coupling effect in an adaptive dipole array," IEEE Transactions on Antennas and Propagation, vol. 52, no. 5, pp.1262-1269, May 2004.

[2] N. Parhizgar, M. A. M. Shirazi, A. Alighanbari, and A. Sheikhi, "Adaptive nulling of a linear dipole array in the presence of mutual coupling," International Journal of RF and Microwave Computer Aided Engineering, vol. 24, pp. 30-38, January 2013.

[3] R. S. Adve and T. K. Sarkar, "Compensation for the effects of mutual coupling on direct data domain adaptive algorithms," IEEE Transactions on Antennas and Propagation, vol. 48, pp. 86-94, January 2000.

[4] K. Kim., T. K. Sarkar, and M. S. Palma, "Adaptive processing using a single snapshot for a nonuniformly spaced array in the presence of mutual coupling and near-field scatterers," IEEE Transactions on Antennas and Propagation, vol. 50, pp. 582590, May 2002.

[5] A. S. C. Svendsen, and I. J. Gupta, "The effect of mutual coupling on the nulling performance of adaptive antennas," IEEE Antennas and Propagation Magazine, vol. 54, no. 3, pp. 17-38, June 2012. 